\title{
Spatial Reuse in IEEE 802.11ax: Whether and How to Use in Practice
}

\author{
Deqing Zhu' ${ }^{*}$ and Shenji Luan ${ }^{2}$ \\ ${ }^{1}$ Academic Affairs Office, Hangzhou Normal University \\ Hangzhou, Zhejiang, 311121, China \\ [e-mail: dqzhu@hznu.edu.cn] \\ ${ }^{2}$ School of Information Engineering, Hangzhou Dianzi University \\ Hangzhou, Zhejiang, 310018, China \\ [e-mail: luanshenji@hdu.edu.cn] \\ *Corresponding author: Deqing Zhu
}

Received March 18, 2021; revised May 5, 2021; accepted May 24, 2021; published December 31, 2021

\begin{abstract}
IEEE 802.11ax is a protocol being developed for high-density Wireless Local Area Networks (WLAN). Several algorithms have been proposed to improve the level of spatial reuse applied in IEEE 802.11ax. However, these algorithms are tentative and do not specify how to select the transmit power and carrier sense threshold in practice; It is unclear when and why the tuned parameters lead to better network performance. In this paper, we restricted the scale of transmit power tuning to prevent the case of backfire in which spatial reuse will result in transmission failure. If the restrictions cannot be satisfied, spatial reuse will be abandoned. This is why we named the proposed scheme as Arbitration based Spatial Reuse (ASR). We quantified the network performance after spatial reuse, and formulate a corresponding maximum problem whose solution is the optimal carrier sense threshold and transmit power. We verified our theoretical analysis by simulation and compared it with previous studies, and the results show that ASR improves the throughput up to 8.6\% compared with 802.11ax. ASR can avoid failure of spatial reuse, while the spatial reuse failure rate of existing schemes can up to $36 \%$. To use the ASR scheme in practice, we investigate the relation between the optimal carrier sense threshold and transmit power. Based on the relations got from ASR, the proposed Relation based Spatial Reuse (RSR) scheme can get a satisfactory performance by using only the interference perceived and the previously found relations.
\end{abstract}

Keywords: IEEE 802.11ax, Spatial reuse, Carrier sense threshold, Transmit power control.

This work was supported by the National Natural Science Foundation of China (No. 61672465), the Zhejiang Provincial National Natural Science Foundation (No. LY17F020024). 


\section{Introduction}

IEEE 802.11ax, also called Wi-Fi 6, is a new Wireless Local Area Networks (WLAN) protocol being designed by IEEE [1][2]. This protocol aims at improving spectrum efficiency and enhancing aggregate throughput of high-density WLAN. In such scenario, the Access Points (APs) and stations are densely deployed in geographically limited environments [3] thus forming a lot of Overlapping Basic Service Sets (OBSS). Interference among those co-channel OBSS may affect spectrum efficiency or cause transmission failure. 802.11ax adopts preamble detection based spatial reuse to overcome this drawback [4].

In the 802.11ax spatial reuse scheme, a node first determines whether a received frame is from OBSS. If it is from OBSS, the node then compares the power of preamble detection with a larger carrier sense threshold called OBSS_PD. If the power is smaller than OBSS_PD, the node will ignore the ongoing transmission and consider the channel is idle. Therefore, the node continues its transmitting procedure, which increases the transmitting opportunity.

Spatial reuse means a certain node ignores the transmissions in OBSS thus may lead to interference increasing. To mitigate the interference, the 802.11ax draft recommends decreasing transmit power if one node has increased its carrier sense threshold. However, some researchers argue that Transmit Power Control (TPC) should not be used because it puts the implementer at a disadvantage. This is represented by Dynamic Sensitivity Control (DSC)[5] scheme. In DSC, only carrier sense threshold is tuned based on the beacon signal power received from AP. Based on DSC, a ratio to balance the adjustment of carrier sense threshold and the transmit power in Balanced Transmit power control and Physical carrier sensing Adaptation (BTPA)[6][7].

However, the above schemes are tentative and do not specify how to select the transmit power and carrier sense threshold in practice. In fact, if transmit power is not properly tuned, the transmissions may fail to meet the Signal to Interference plus Noise Ratio (SINR) requirement thus result in transmission failure, which is the backfire of spatial reuse. Hence, it is significant to determine how to tune these parameters in practice and evaluate the network performance after spatial reuse. This is the motivation of this paper. The main contributions are

1) We devised an Arbitration based Spatial Reuse (ASR) scheme applied in IEEE 802.11ax. In ASR, spatial reuse will be abandoned when the minimum allowed transmit power $\left(P_{\text {TXmin }}\right)$ is bigger than the maximum allowed transmit power $\left(P_{\mathrm{TXmax}}\right)$. Specifically, the transmit power of node $e_{\text {ai }}$ (the node which attempts to initiate spatial reuse) must be strong enough to let the SINR at its AP exceed the given threshold, which defines $P_{\text {TXmin }}$. On the other hand, the transmit power must be not so strong to corrupt the ongoing transmissions in OBSS, which gives $P_{\text {TXmax }}$. The transmit power tuning is restricted between $P_{\text {TXmin }}$ and $P_{\text {TXmax }}$, which prevents the backfire of spatial reuse.

2) We devised a simple but still accurate way to evaluate the network performance after spatial reuse. Based on this, we formulate an aggregate throughput maximum problem whose solution leads to the optimal transmit power and carrier sense threshold.

3) We validated our theoretical analysis by simulation. Compared with 802.11ax, ASR improves the throughput up to $8.6 \%$. ASR can avoid failure of spatial reuse, while the spatial reuse failure rate of existing schemes can up to $36 \%$. 
4) We explored the relation between the optimal sensing threshold and transmit power. In practice, we can just use the relation and the perceived interference to get a close-to-optimal network performance.

The rest of this paper is organized as follows. In Section 2, we give an overview of the related work which aims to improve the level of spatial reuse. In Section 3, we describe the proposed scheme and its theoretical base. In Section 4, we verify theoretical analysis by simulation and compared the proposed scheme with existing work. Following that, we conclude this paper in Section 5.

\section{Related Work}

Research efforts have been dedicated to improving the level of spatial reuse. The impact of the SINR and node topology on the optimal carrier sense threshold was studied in ad hoc networks[8]. The authors in [9] proposed a carrier sense threshold adaptation method for better spatial reuse, which considered both interferer and destination nodes. TPC is also recognized as a way to improve spatial reuse. In [10], the authors showed that TPC can improve the channel efficiency in ad hoc networks. It is also proved that TPC offers several advantages that cannot be achieved by tuning the carrier sense threshold[11]. The researchers in [12] tuned the transmit power and carrier sense threshold jointly according to different interference types to improve aggregate throughput. However, the above schemes focus on ad hoc multi-hop networks or early 802.11 protocols and thus cannot be directly applied in 802.11ax.

Several algorithms have been proposed to improve the level of spatial reuse for IEEE 802.11ax. A way to calculate the transmit power $P_{T X a x}$ based on preamble detection is given[13]:

$$
\begin{gathered}
P_{\text {TXax }}=P_{\text {TXref }}-\left(O B S S_{-} P D-O B S S_{-} P D_{\min }\right), \\
O B S S_{-} P D_{\min } \leq O B S S_{-} P D \leq O B S S_{-} P D_{\max },
\end{gathered}
$$

where $P_{\text {TXref }}$ is the reference transmit power, $O B S S_{-} P D_{\min }$ and $O B S S_{-} P D_{\max }$ is the minimum and maximum value of OBSS_PD. The Dynamic Sensitivity Control (DSC)[5] scheme does not use TPC and only tunes its OBSS_PD as

$$
O B S S_{-} P D_{d s c}=P_{R X}^{A P}-M,
$$

where $P_{R X}^{A P}$ is the beacon signal power received from AP and $M$ is a margin integer. The BTPA [6][7] adapt a ratio $\theta$ to jointly tune the carrier sense threshold and transmit power. That is

$$
\begin{aligned}
\Delta x[d B]= & O B S S_{-} P D_{d s c}-O B S S_{-} P D_{\min }, \\
P_{T X b t p a} & =P_{\mathrm{TXdft}}-\theta \Delta x, \\
O B S S_{-} P D_{b t p a} & =O B S S_{-} P D_{d s c}+\Delta x(1-\theta),
\end{aligned}
$$

where $P_{\text {TXdft }}$ is a node's default transmit power. The performance of DSC and IEEE 802.11ax draft have been thoroughly studied [14][15][16]. However, these algorithms are tentative and not clear when and why the tuned parameters lead to better network performance thus hard can be used in practice. In this paper, we quantified the network performance after spatial reuse to find the relation between the optimal transmit power and carrier sense threshold. In practice, 
we only use the perceived interference and the relations and can get a satisfactory performance.

\section{Arbitration based Spatial Reuse (ASR)}

For a chosen carrier sense threshold, the bound of transmit power tuning should be restricted to prevent the backfire of spatial reuse. To find the optimal carrier sense threshold and transmit power, we quantified the network performance after spatial reuse.

\subsection{Restrict transmit power to prevent backfire}

The transmit power tuning should be confined to prevent the case of backfire in which spatial reuse will result in degraded network performance. The transmit power of node $e_{\text {ai }}$ (the node which attempts to initiate spatial reuse) must satisfy two restrictions. One is that the transmit power must ensure its desired receiver can sustain a required SINR, i.e. the transmit power has a lower bound. The other is the interference induced by node $e_{\mathrm{ai}}$ must not deprive the ongoing co-channel transmissions of sustaining their required SINR, i.e. the transmit power has an upper bound.

\subsubsection{The minimum allowed transmit power}

Note that the desired signal for a specific receiver will be regarded as interference by other co-channel receivers. Thus the SINR of the receiver with the desired transmitter is defined as

$$
S I N R=\frac{\bar{P}_{R X}^{T X}}{\sum_{i \in O B S S} \bar{P}_{R X}^{T X_{i}}},
$$

where $\bar{P}_{R X}^{T X}$ is the received power (in $m w$ ) at receiver node-RX from the desired transmitter node-TX. $\sum_{i \in O B S S} \bar{P}_{R X}^{T X_{i}}$ is the total received power (interference) from the ongoing transmissions of co-channel OBSS.

The received power and interference will be express hereinafter in $d B m$, namely, $P_{R X}^{T X}=10 \log _{10}\left(\bar{P}_{R X}^{T X}\right)$.

The minimum transmitting power of node $e_{\text {ai }}$ must meet the SINR requirement of its receiver (denoted by $\mathrm{AP}_{0}$ ). We can rewrite the received power and interference of (6) in $\mathrm{dBm}$ as

$$
\begin{aligned}
& 10 \log _{10} \bar{P}_{A P_{0}}^{\text {node }_{\text {ai }}}-10 \log _{10} \sum_{i \in O B S S} \bar{P}_{A P_{0}}^{T X_{i}} \\
& =P_{A P_{0}}^{\text {node }} e_{\text {ai }}-10 \log _{10} \sum_{i \in \text { OBSS }} 10^{P_{A X_{0}}^{T X_{i}} / 10}>\beta,
\end{aligned}
$$

where $\beta$ is the SINR requirement at $\mathrm{AP}_{0}$.

The received power at a receiver from the desired transmitter is defined as (in $\mathrm{dBm}$ ) [18]

$$
P_{R X}^{T X}=P_{T X}+G-P L-P n L o s s
$$

where $P_{T X}$ is a node's transmit power and $G$ is the antenna gain of the transmitter in $d B i$, PnLoss is the penetration loss due to cross BSS wall in $d B, P L$ is the path loss due to traveling over a distance or obstacles in-between [18]. The path loss model used in this paper is the one developed for IEEE802.11n known as the "Erceg" model [17], namely: 


$$
P L=\left\{\begin{array}{ll}
L_{F S}(d), & \text { if } d \leq d_{B P} \\
L_{F S}\left(d_{B P}\right)+35 \log _{10}\left(\frac{d}{d_{B P}}\right), & \text { if } d>d_{B P}
\end{array},\right.
$$

where $d$ is the distance between the transmitter and the receiver, $d_{\mathrm{bp}}$ is the "breakpoint distance" for indoor office. $L_{\mathrm{FS}}$ is free space loss and $L_{F S}(d)=40.05+20 \log _{10}\left(\frac{f c}{2.4}\right)+20 \log _{10}(d)$, where $f_{\mathrm{c}}$ is the bandwidth in GHz.

Using (7) and (8), the minimum allowed transmit power of node $e_{\text {ai }}$ can be expressed as (in $d B m)$

$$
P_{T X \min }=\beta-G+P L_{a i-A P_{0}}+10 \log _{10} \sum_{i \in O B S S} 10^{P_{A P_{0}}^{T X_{i}} / 10},
$$

where $P L_{a i-A P_{0}}$ is the path loss between node ai and $A P_{0}$ calculated by (9). Similar to [19], we let $\mathrm{AP}_{0}$ announce the values of its current interference, node $e_{\text {ai }}$ then use (10) to calculate $P_{\text {TXmin }}$.

\subsubsection{The maximum allowed transmit power}

The maximum transmitting power of node $e_{\mathrm{ai}}$ must not corrupt the ongoing transmissions in OBSS. Similar to [19], we let each receiving AP in OBSS announce the values of their desired signal and current interference, node $e_{\text {ai }}$ then calculate the SINRs of these APs after adding the interference caused by itself. Process similarly, the SINR at $A P_{\mathrm{i}}$ must meet the requirement:

$$
P_{A P_{i}}^{T X_{i}}-10 \log _{10}\left(10^{P_{A P_{i}}^{\text {nodeai }} / 10}+10^{\text {Itf obss }} / 10+10^{\psi / 10}\right)>\beta,
$$

where $P_{A P_{i}}^{\text {node }}$ ii the interference at each receiving AP of OBSS caused by node $e_{\text {ai. }}$ It $f_{\text {obss }}$ is caused by transmission in other OBSS depicted in Fig. 1 while $\Psi$ is caused by transmission belonging to the first tier co-channel OBSS of $A P_{\mathrm{i}}$ but not shown in Fig. 1. Using (8) substitute $P_{A P_{i}}^{\text {node }_{a i}}$ into (11), we can get

$$
\begin{aligned}
& P_{T X \max }=\min \left(P L_{a w-A P_{i}}+2 \text { PnLoss }-G+10 \log \left(\left(\frac{P_{A P_{i}}^{T X_{i}}-\beta}{10}\right)\right.\right. \\
& \left.-\left(10^{\frac{\psi}{10}}+10^{\frac{I t f_{\text {obss }}}{10}}\right)\right) .
\end{aligned}
$$

If $P_{T X \text { min }}>P_{T X \text { max }}$, that means spatial reuse will result in transmitting failure. Therefore, spatial reuse should not be used.

\subsection{Quantify the network performance after spatial reuse}

To find the optimal carrier sense threshold and transmit power, we devised a method to quantify the network performance after spatial reuse.

The network architecture used is shown in Fig. 1 where each regular hexagon represents a Basic Service Set (BSS). Because there are only 3 available channels available when IEEE 802.11 protocol operating in $2.4 \mathrm{GHz}$, the central hexagon together with those hexagons in grey shadow share the same channel. As pointed out in [8], the interference of the central hexagon is mainly contributed by the first-tier co-channel BSS, i.e. the hexagons in grey shadow. Therefore, spatial reuse will be conducted among a given BSS and its first-tier co-channel BSS. Next, we introduce several notions. As illustrated in Fig. 1, the Carrier sensing Area (CA) for STA 1 is the dotted circle area with $d_{\mathrm{cs}}$ as its radius and the node itself as 
its center. The Hidden node Area (HA) is defined as the remaining area of the central hexagon excluding the CA. The Spatial reuse Occupy Area (SOA) is defined as the overlapping of the co-channel BSS and the CA. STA 1 could initiate a transmission only when the ongoing

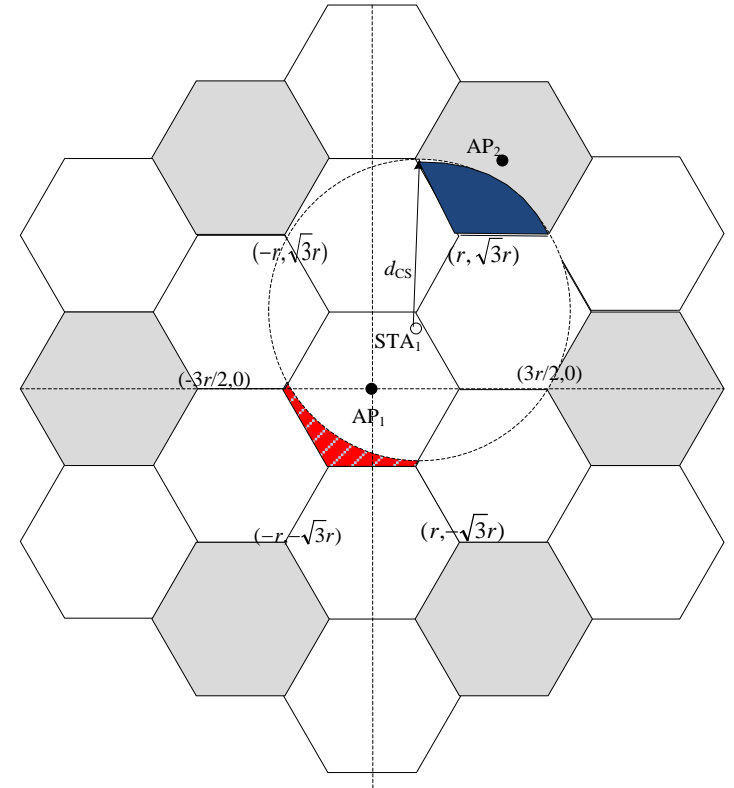

Fig. 1. Network Architecture.

transmissions are not in SOA. As shown in Fig. 1, the HA and SOA of STA 1 is the area in red and the area in dark blue shadow, respectively.

According to the conclusion of [21], a node's transmit probability $\tau$ in a given slot is between $2 /(W+1)$ and $2 /\left(1+2^{N} W\right)$, where $W$ is the maximum contention window under CSMA/CA, and $N$ is the permitted maximum retransmission. Assume that there are $h$ nodes in each BSS, the probability that a successful transmission is

$$
p=h \tau(1-\tau)^{h-1} .
$$

Whether node ai can initiate a transmission is determined by two factors. Firstly, there is no node transmit in the HA. Since there are $h$ nodes in each BSS, the number of nodes in HA is round $\left(\frac{s_{h}}{s_{B S S}} h\right)$, where "round" the function taking the most approximate integer. Therefore, let $\zeta$ be the probability that there is no transmission in HA when node $e_{\text {ai }}$ initiates a transmission, we have

$$
\begin{aligned}
& \zeta=(1-\tau)^{\operatorname{round}\left(\frac{s_{h}}{s_{B S S}} h\right)}\left(h-\operatorname{round}\left(\frac{s_{h}}{s_{B S S}} h\right)\right) \tau(1-\tau)^{\left(h-\operatorname{round}\left(\frac{s_{h}}{s_{B S S}} h\right)-1\right)} \\
& =\left(h-\operatorname{round}\left(\frac{s_{h}}{s_{B S S}} h\right)\right) \tau(1-\tau)^{(h-1)} \\
& \approx\left(1-\frac{s_{h}}{s_{B S S}}\right) h \tau(1-\tau)^{(h-1) .}
\end{aligned}
$$

Secondly, there is no node transmitting in SOA. Similarly, we can get 


$$
\eta=\prod_{i=1}^{6}\left(1-\frac{s(i)}{s_{B S S}} h \tau(1-\tau)^{h-1}\right)
$$

See section Appendix for how to calculate $s_{\mathrm{h}}$ and $s(i)$ in detail.

Using Shannon formula, the aggregate throughput can be expressed proportional to channel rate, that is

$$
R_{T} \propto \xi \eta \log _{2}\left(1+\operatorname{SINR}_{a i}\right)+\sum_{i=1}^{6} \log _{2}\left(1+\operatorname{SINR}_{i}\right),
$$

where $S I N R_{\mathrm{ai}}$ is the SINR of $A P_{0}$, and $S I N R_{\mathrm{i}}$ is the SINR of $A P_{\mathrm{i}}$. Thus, we can formulate the throughput maximization problem

$$
\begin{aligned}
& \max \left(R_{T}\right) \\
& \text { w.r.t } \quad d_{c S}, \quad P_{T X}, \\
& \text { s.t. } \quad P_{T X \text { min }}<P_{T X \text { max }} .
\end{aligned}
$$

where $d_{\mathrm{cs}}$ is sensing range (see section Appendix for the relation between $d_{\mathrm{cs}}$ and the carrier

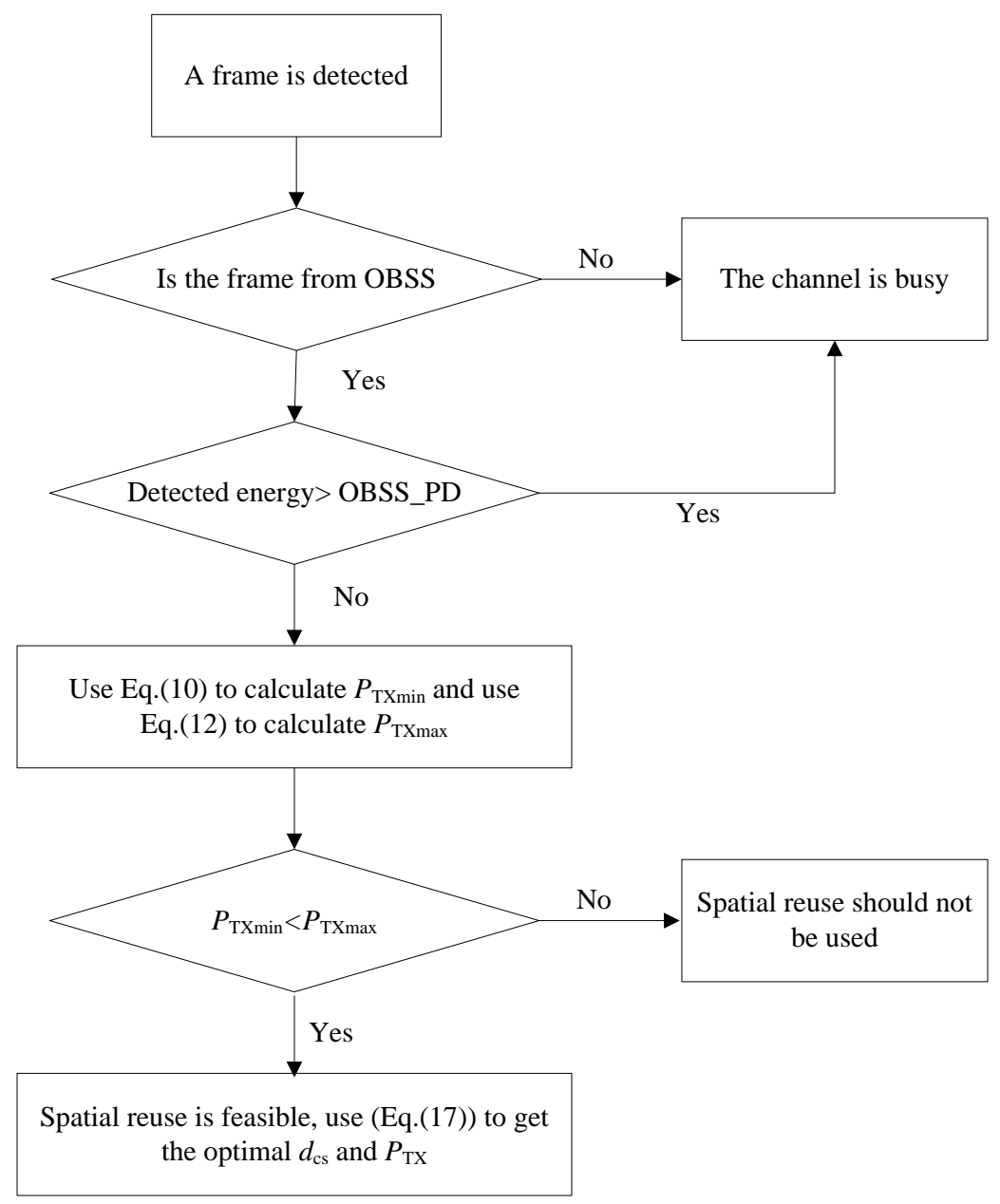

Fig. 2. Diagram of the proposed ASR. 
sense threshold ). Note that the carrier sense threshold is confined between $-82 \mathrm{dBm}$ and $-62 \mathrm{dBm}$, and the transmit power of a node is between $1 \mathrm{dBm}$ and $15 \mathrm{dBm}$, the optimal carrier sense threshold and transmit power thus can be easily found by using exhaustive search.

\subsection{The ASR scheme}

Our ASR scheme contains the steps as shown in Fig. 2. The node ai gets the interference information from the AP it associated and then use (10) to calculate $P_{\mathrm{TXmin}}$. It receives the interference information broadcasted by the AP in OBSS and use (12) to calculate $P_{\text {TXmax }}$. Only when $P_{T X \text { min }} \leq P_{T X \text { max }}$, spatial reuse is feasible. Otherwise, spatial reuse should not be implemented.

\section{Performance Analysis}

\subsection{Validation via simulation}

To verify our theoretical analysis, the following experiments are implemented using MATLAB m-code programming language. For theoretical analysis, we need to generate the positions of node $_{\text {ai }}$ and nodes which are transmitting in OBSS. To generate the node $e_{\text {ai }}$, we first generate randomly the positions of $h$ nodes in the regular hexagon area, then all the nodes are polled using the method as follows. A random number between 0 and 1 is generated, and if it is smaller than $\tau$, then the node will be considered as a potential transmitter. If there is only one potential transmitter in the BSS, then this node is node $e_{\text {ai }}$ and its position is recorded. Next, we generate the positions of transmitting nodes in OBSS. For each OBSS, using a similar way to determine whether there is a transmitting node. If there exists a transmitting node and it is the first transmitting node generated in OBSS, the position can be randomly chosen. However, for the other transmitting nodes in OBSS, the interference caused by already existed transmitting nodes should be considered. The position of the new transmitting node must meet the SINR requirement of the already existed node in OBSS, i.e. the following equation must be satisfied

$$
P_{A P_{i}}^{T X_{i}}-10 \log _{10}\left(10^{I t f_{\text {obss }} / 10}+10^{\psi / 10}\right)>\beta,
$$

where the parameters are the same as (11). If (18) cannot be satisfied, the position of the newly generated transmitting node should be changed. If the retry of changing position exceeds 10 we then assume no transmitting node in the BSS. After node $e_{\text {ai }}$ and all transmitting nodes have been generated, then we can use (17) to calculate the maximum $R_{\mathrm{T}}$.

For simulation, we first calculate the distance of node $e_{\text {ai }}$ and other nodes in the same BSS based on nodes generated in theoretical analysis, if the distance is bigger than $d_{\mathrm{cs}}$, it is considered as a hidden node. Use a similar way, only nodes which are not hidden node was polled to determine whether exist a successful transmitting node, if it does, we then polling the hidden nodes, if any hidden node will transmit, it will corrupt node $e_{\mathrm{ai}}$, which means there is no node $_{\mathrm{ai}}$ in the simulation scenario. If the distance between node $e_{\mathrm{ai}}$ and the transmitting node in OBSS is smaller than $d_{\mathrm{cs}}$, node $e_{\mathrm{ai}}$ cannot transmit, i.e. in this case, there is no node $e_{\mathrm{ai}}$.

The parameters used in theoretical calculation and simulation are listed in Table 1. These values are based on the suggestion evaluation method of 802.11ax[18]. To see the difference 
between theoretical and simulation results, we define the function $\operatorname{Err}=\frac{\left|R_{T c a l c u}-R_{T s i m u}\right|}{R_{T c a l c u}}$.

Table 1. The parameters used in calculation and simulation

\begin{tabular}{|c|c|c|c|}
\hline parameter & value & parameter & value \\
\hline \hline$W$ & 31 & $G / \mathrm{dBi}$ & -2 \\
\hline$N$ & 3 & $P n L o s s / \mathrm{dB}$ & 3 \\
\hline$r / \mathrm{m}$ & 10 & $d_{B P} / \mathrm{m}$ & 10 \\
\hline$P D_{\min } / \mathrm{dBm}$ & -82 & $P_{T X r e f} / \mathrm{dBm}$ & 21 \\
\hline$P D_{\max } / \mathrm{dBm}$ & -62 & $T_{\mathrm{CSdft}} / \mathrm{dBm}$ & -82 \\
\hline$P_{\mathrm{TXdft}} / \mathrm{dBm}$ & 15 & $P_{\mathrm{dftAP}} / \mathrm{dBm}$ & 20 \\
\hline
\end{tabular}

The average of 3000 times results is depicted in Fig. 3. We can see that the simulation results consistent with the theoretical one, which verifies our analysis model.
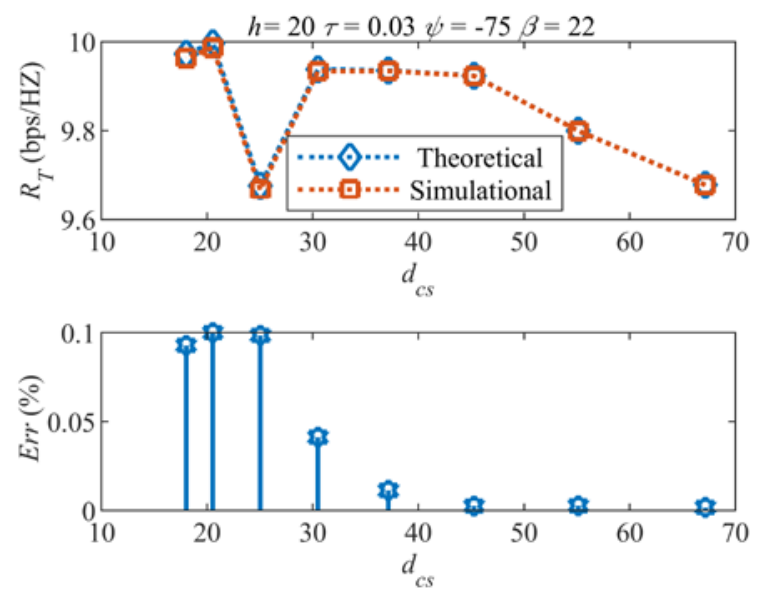

(a) when $\tau=0.03$
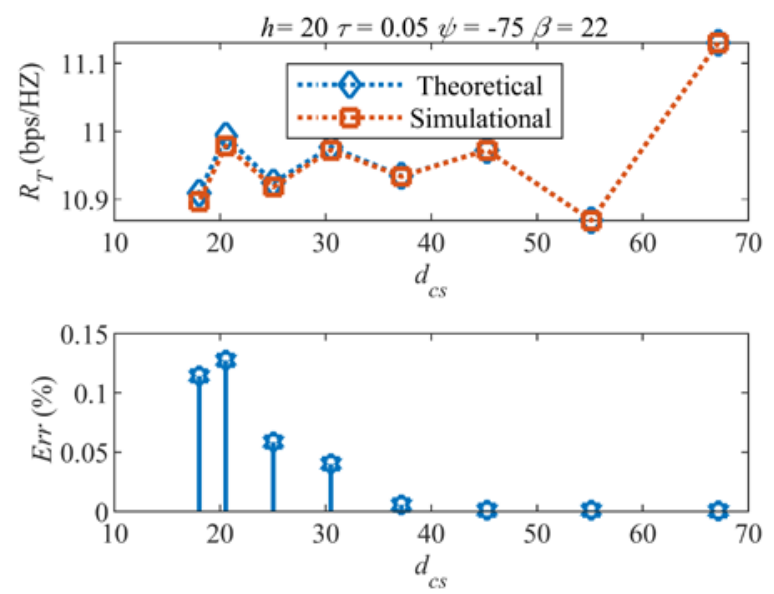

(b) when $\tau=0.05$

Fig. 3. Theoretical and simulation results as $d_{\mathrm{cs}}$ varies 


\subsection{Compared with existing work}

\subsubsection{Aggregate throughput}

The aggregate throughput achieved by each scheme is shown in Fig. 4. As can be seen, when the transmit probability $\tau$ is small, all the schemes achieve almost the same throughput (Fig. 4 (a)). This is because few nodes will transmit, and there is little chance to resort to spatial reuse. The same case can be seen when the number of nodes $h$ is small (Fig. 4 (c)).

As the number of nodes or transmit probability of each node increases, the performance advantages of our scheme over other schemes becomes notable. Our scheme outperforms other existing schemes when more concurrent transmissions exist, or interference level is higher or the SINR requirement is high. This is because our ASR scheme adopts the arbitration mechanism, which means node $e_{\mathrm{ai}}$ keeps the information of the minimum and maximum allowed transmit power, and based on the information, whether transmit or not is decided. While in other schemes the transmission of node ai may ruin concurrent transmission in OBSS and itself. Note that the scenarios in which our scheme outperforms other existing schemes are exactly the scenarios that the IEEE 802.11ax operates.

As can be seen from Fig. 4, DSC gets the lowest performance in most cases, and our ASR outperforms BTPA notably. This is because the power of beacons cannot accurately reflect the interference level of OBSS, thus makes it hard to set a proper carrier sense threshold and transmit power.

As shown in Fig. 4, the schemes with TPC achieve a better throughput in almost every case. This is because decreasing the carrier sense range to enable spatial reuse may corrupt the ongoing co-channel transmissions. The situation gets even worse in DSC due to it always uses the maximum transmit power. As pointed out by some researchers, TPC will make the node in a disadvantage position, i.e. the nodes not applied TPC will benefit because they are less bothered by the transmissions of that node. However, if we want to increase the level of spatial reuse, reducing transmit power thus to mitigate interference might be a reasonable choice.

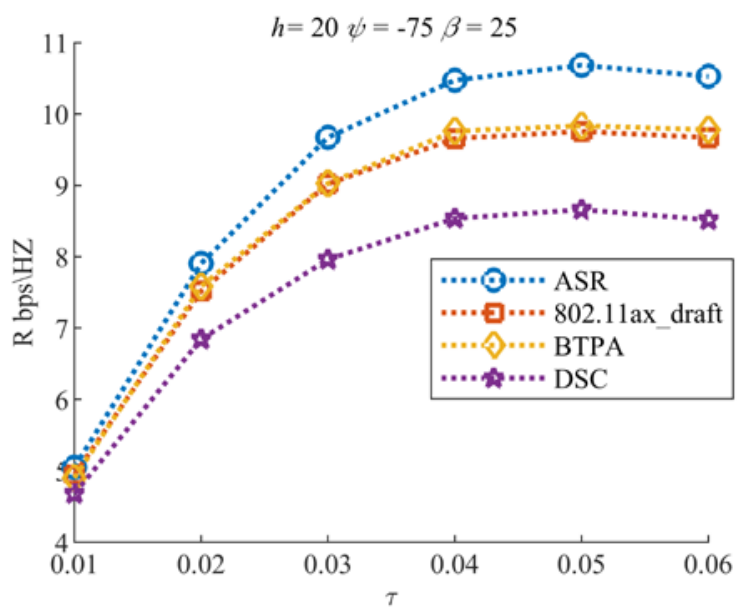

(a) Varying of $\tau$ 


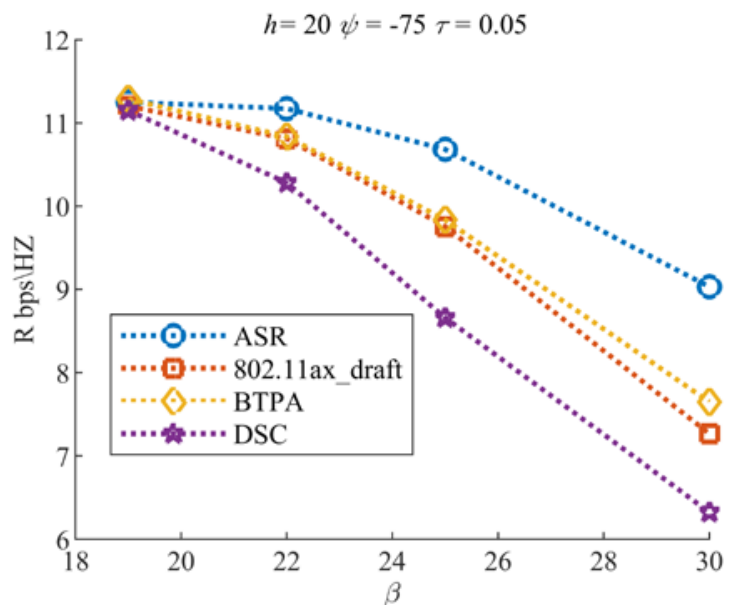

(b) Varying of $\beta$

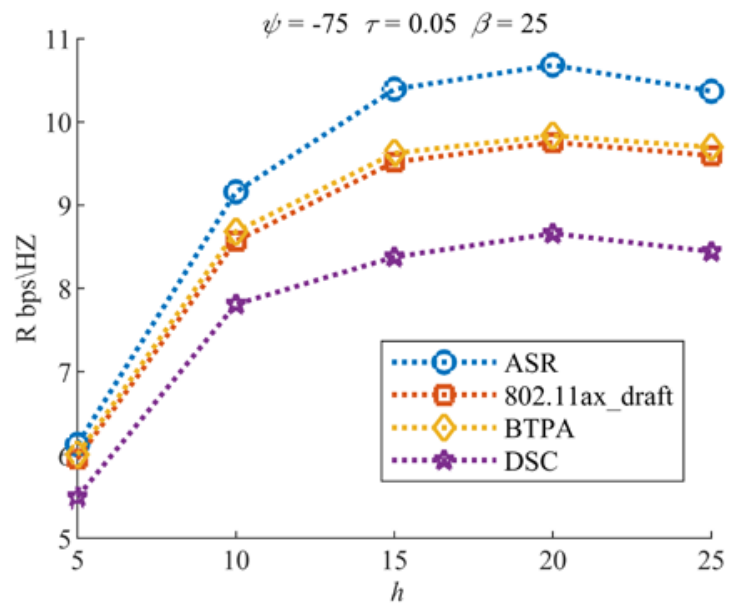

(c) Varying of $h$

Fig. 4. The aggregate throughput comparison

\subsubsection{Spatial reuse failure rate}

We define the metric called Spatial Reuse Failure Rate (SRFR), which is the proportion of spatial reuse that result in transmission failure of node $e_{\text {ai }}$ or the OBSS transmitters. Since the transmit power in our scheme is restricted, the SINR in our scheme meets the requirement. Thus, no SRFR is produced in ASR whereas the existing works may produce SRFR, i.e., our scheme surely outperforms the existing works in terms of SRFR. As can be seen from Fig. 5 the SRFR of existing work may up to $36 \%$.

\subsection{Where can we expect the best network performance}

Since we are tuning the carrier sense threshold and transmit power, it naturally leads to the following questions: 1 ) where can we expect the maximum aggregate throughput, 2) is there any relation between the optimal carrier sense threshold and transmit power? 


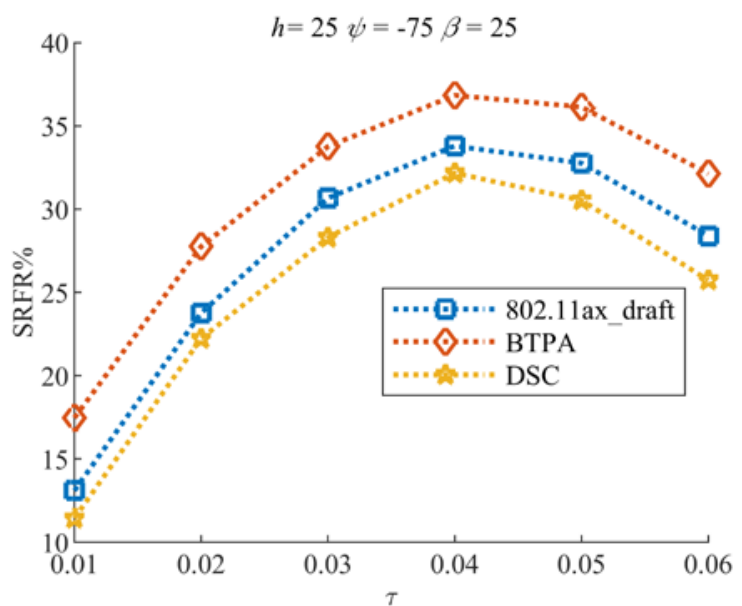

Fig. 5. Spatial reuse failure rate of existing work

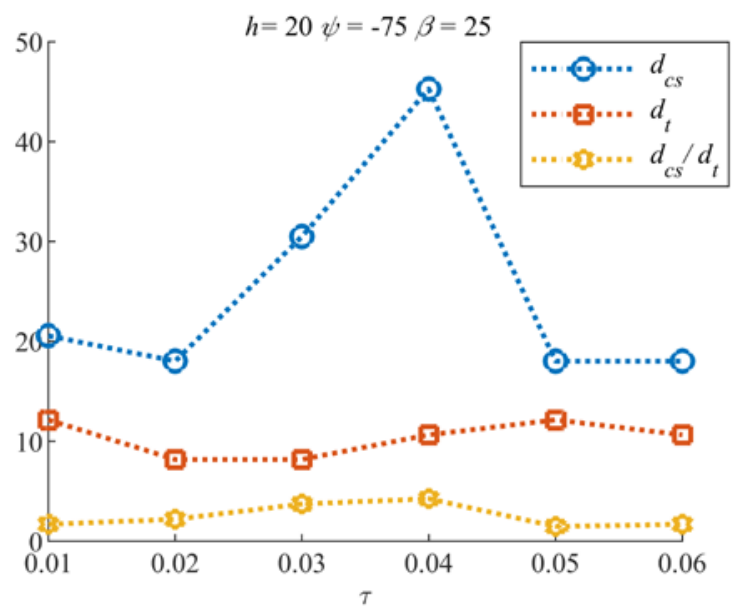

Fig. 6. The relations of optimal $d_{\mathrm{cs}}, d_{\mathrm{t}}$, and $d_{\mathrm{cs}} / d_{\mathrm{t}}$

Note that carrier sense threshold and transmit power are not in the same dimension, we need to transform them into measured by meters. Based on (8) and (9), the transform of transmit power and transmit range can be got by

$$
d_{t}= \begin{cases}10 \frac{\left(P_{\mathrm{TX}}-40.05+\mathrm{G}+I_{\text {total }}-\beta\right)}{20}, & \text { if } P_{\mathrm{TX}} \leq 2.05 \\ 10 \times 10 \frac{\left(P_{\mathrm{TX}}-66.05+\mathrm{G}+I_{\text {total }}-\beta\right)}{35} & \text {,if } P_{\mathrm{TX}}>2.05 .\end{cases}
$$

where $I_{\text {total }}$ is total interference received by the transmitter. Because the value of $I_{\text {total }}$ is changing with time, we assume that $I_{\text {total }}$ is $-82 \mathrm{dBm}$ and $\beta$ is $22 \mathrm{~dB}$ for a unified mapping. Combined with (8) and (9), the threshold 2.05 in (19) can be got. Fig. 6 shows the relations of optimal $d_{\mathrm{cs}}, d_{\mathrm{t}}$, and $d_{\mathrm{cs}} / d_{\mathrm{t}}$ as $\tau$ increases. It gives us a direct impression that where can we expect the optimal $R_{\mathrm{T}}$.

\subsection{How to use ASR in practice}

Since the relations in Fig. 6 are got by average, it's reasonable to believe that the relation will approximately the same for the given $h, \tau, \beta$, and $\Psi$. Therefore, we designed a tuning scheme based on the relation of $d_{\mathrm{cs}} / d_{\mathrm{t}}$. We first need to determine the sensing threshold or transmit 
power. Note that we can use the total inference perceived by node $e_{\text {ai }}$ as its sensing threshold and the corresponding sensing range, then use the relation we can get the transmit range. Finally, the transmit power can be got by

$$
P_{\text {TXrelation }}=\left\{\begin{array}{c}
I_{\text {total }}+\beta-G+40.05+20 \log _{10}\left(d_{t}\right), \\
\text { if } d_{t} \leq 10 ; \\
I_{\text {total }}+\beta-G+66.05+35 \log _{10}\left(\frac{d_{t}}{10}\right), \\
\text { if } d_{t}>10 .
\end{array}\right.
$$

When spatial reuse is feasible, the RSR scheme follows the following steps rather than calculate the optimal $d_{\mathrm{cs}}$ and $P_{\mathrm{TX}}$ using (17)

Step1. node $e_{\text {ai }}$ use the total inference perceived as its sensing threshold, thus get $d_{\mathrm{cs}}$.

Step2. Based on the saved relation of $d_{\mathrm{cs}} / d_{\mathrm{t}}$, node ai get $d_{\mathrm{t}}$, and thus set the corresponding transmit power using (20).

Fig. 7 depicts the performance of RSR scheme. As can be seen, the performance of RSR is the one closest to the optimal. Though the scheme is very simple, it can get nearly optimal $R_{\mathrm{T}}$.

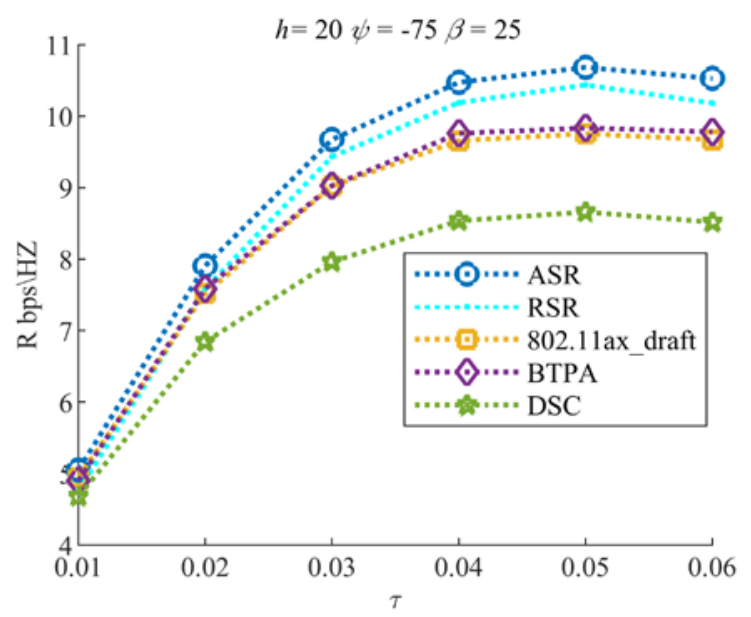

(a) Varying of $\tau$

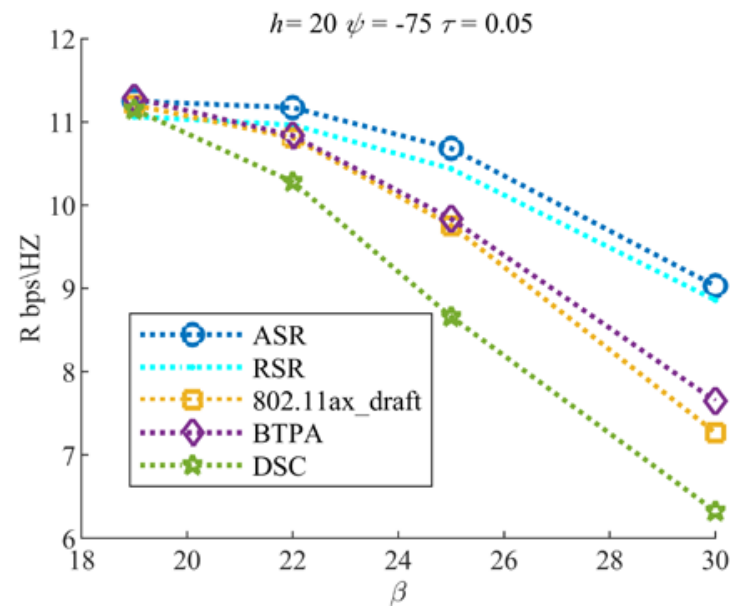

(b) Varying of $\beta$ 


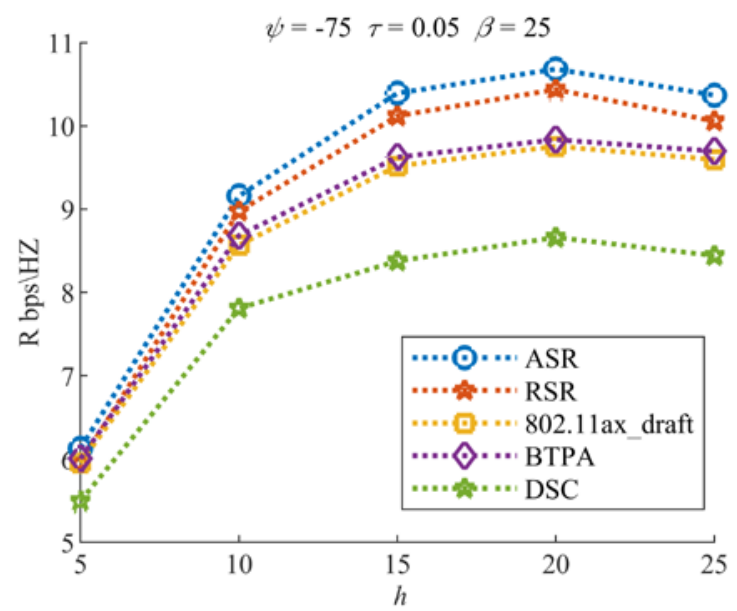

(c) Varying of $h$

Fig. 7. The performance of RSR scheme

\section{Conclusions}

In this paper, we devised an arbitration based scheme in which spatial reuse will be abandoned if the transmit power restrictions are not met. We quantified the network performance after spatial reuse to determine the optimal carrier sense threshold and transmit power. Comparison results show that our scheme improves the throughput up to $8.6 \%$ and can avoid the backfire of spatial reuse. We proposed a relation based scheme that only needs two parameters: the received interference and the saved relations, but it really got a satisfactory performance. We believe that the scheme could be used in practice. Although we focus only on the uplink scenario, the proposed schemes can be easily expanded to the downlink or combined scenario.

\section{References}

[1] Afaqui M S, Garcia-Villegas E, Lopez-Aguilera E, "IEEE 802.11 ax: Challenges and requirements for future high efficiency WiFi,” IEEE Wireless Communications, vol. 24, no. 3, pp. 130-137, Dec. 2017. Article (CrossRef Link)

[2] Khorov E, Kiryanov A, Lyakhov A, et al, "A tutorial on IEEE 802.11 ax high efficiency WLANs," IEEE Communications Surveys \& Tutorials, vol. 21, no. 1, pp. 197-216, Sep. 2018.

Article (CrossRef Link)

[3] Kamel M, Hamouda W, Youssef A, "Ultra-dense networks: A survey," IEEE Communications Surveys \& Tutorials, vol. 18, no. 4, pp. 2522-2545, May 2016. Article (CrossRef Link)

[4] Considerations for Spatial Reuse, IEEE Standard 11-15-1313, 2015. Article (CrossRef Link)

[5] DSC Proposal Text, IEEE Standard 11-16-0310, 2016. Article (CrossRef Link)

[6] Jamil I, "Improving spatial reuse in future dense high efficiency Wireless Local Area Networks," Ph.D. dissertation, Doctoral School mathematics, telecommunications, computer science, signal, systems, electronics, Rennes INSA, Rennes, France, 2015. Article (CrossRef Link)

[7] Spatial Reuse DSC and TPC, IEEE Standard 11-18-1531, 2018. Article (CrossRef Link)

[8] Zhai H, Fang Y, "Physical carrier sensing and spatial reuse in multirate and multihop wireless ad hoc networks," in Proc. of INFOCOM, Barcelona, Spain, pp.1-12, 2006. Article (CrossRef Link)

[9] Kim S, Yoo S, Yi J, et al, "FACT: Fine-grained adaptation of carrier sense threshold in IEEE 802.11 WLANs," IEEE Transactions on Vehicular Technology, vol. 66, no. 2, pp. 1886-1891, Feb. 2017. Article (CrossRef Link) 
[10] Monks J P, Bharghavan V, Hwu W, "A power controlled multiple access protocol for wireless packet networks,” in Proc. of INFOCOM, AK, USA, pp.219-228, 2001. Article (CrossRef Link)

[11] Kim T, Lim H, Hou J, "Improving spatial reuse through tuning transmit power, carrier sense threshold, and data rate in multihop wireless networks," in Proc. of MobiCom, Los Angeles CA USA, pp.366-377, 2006. Article (CrossRef Link)

[12] Ma H, Zhu J, Roy S, et al, "Joint transmit power and physical carrier sensing adaptation based on loss differentiation for high density IEEE 802.11 WLAN," Computer Networks, vol. 52, no. 9, pp. 1703-1720, Jun. 2008, Article (CrossRef Link)

[13] OBSS_PD/TPC Examined, IEEE Standard 11-17-0582, 2017. Article (CrossRef Link)

[14] Selinis I, Filo M, Vahid S, et al, "Evaluation of the DSC algorithm and the BSS color scheme in dense cellular-like IEEE 802.11 ax deployments," in Proc. of PIMRC, Valencia, Spain, pp.1-7, 2016. Article (CrossRef Link)

[15] Selinis I, Katsaros K, et al, "Control OBSS/PD sensitivity threshold for IEEE 802.11 ax BSS color," in Proc. of PIMRC, Bologna, Italy, pp.1-7, 2018. Article (CrossRef Link)

[16] Iwata M, Yamamoto K, Yin B, et al, "Analysis of Inversely Proportional Carrier Sense Threshold and Transmission Power Setting Based on Received Power for IEEE 802.11 ax," in Proc. of CCNC, Las Vegas, USA, pp.1-6, 2019. Article (CrossRef Link)

[17] IEEE 802.11ax Channel Models, IEEE Standard 11-14-0882, 2015. Article (CrossRef Link)

[18] TGax Evaluation Methodology, IEEE Standard 11-14-0571, 2016. Article (CrossRef Link)

[19] Monks J P, Bharghavan V, Hwu W, "A power controlled multiple access protocol for wireless packet networks,” in Proc. of IEEE INFOCOM, Anchorage, AK, pp. 219-228, 2001. Article (CrossRef Link)

[20] Merlin S, Merlin S, Sampath H, et al., TGax Simulation Scenarios, IEEE Standard 11-14-0980, 2015. Article (CrossRef Link)

[21] Bianchi G, "Performance analysis of the IEEE 802.11 distributed coordination function," IEEE Journal on Selected Areas in Communications, vol. 18, no. 3, pp. 535-547, Mar. 2000.

Article (CrossRef Link)

\section{Appendix}

1)The relation between carrier sense threshold and carrier sense range

For a certain carrier sensing threshold $T_{\mathrm{cs}}$, there is a corresponding sensing range $d_{\mathrm{cs}}$ which in turn determines the HA and SOA. We first show how to convert $T_{\mathrm{cs}}$ to $d_{\mathrm{cs}}$. Recall that sensing range is defined as how far a transmission can be heard with signal strength exceeds $T_{\text {cs }}$. Assume that when the sensing range is smaller than the breakpoint distance $d_{\mathrm{BP}}$, one (otherwise two) cell wall penetration loss will be taken into account. Using (8) and (9), the relation between $T_{\mathrm{cs}}$ and $d_{\mathrm{cs}}$ is

$$
d_{c s}=\left\{\begin{array}{cc}
10 \frac{\left(\frac{\left.P_{\mathrm{dft}}+G-40.05-\text { PnLoss- } T_{c s}\right)}{20},\right.}{20}, & T_{c s}>-47.05 \mathrm{dBm} ; \\
d_{\mathrm{BP}} 10^{\left(\frac{\left(\mathrm{P}_{\mathrm{dft}}+G-60.05-2 \text { PnLoss- } T_{c s}\right)}{35},\right.}, & T_{c s}<-47.05 \mathrm{dBm} .
\end{array}\right.
$$

where $P_{\mathrm{dft}}$ is the default transmit power, the value -47.05 can be got when the transmission distance is equal to $d_{\mathrm{BP}}$, with parameter values in Table $\mathbf{1}$ [21].

2)The relation of carrier sense range, $H A$, and $S O A$

Next, we will show how to determine HA and SOA with known $d_{\text {cs. }}$ Let $r$ denote the side length of the regular hexagon, node $e_{\text {ai }}$ denote the node which attempts to initiate a transmission, and $(x, y)$ be the coordinates of node $e_{\text {ai }}$. Let $d_{\mathrm{i}}(i=1, \cdots 6)$ be the distance between node $e_{\mathrm{ai}}$ and the nearest vertex of each co-channel OBSS . With the corresponding coordinates of the vertexes shown in Fig. 1, we have 


$$
d_{i}=\sqrt{(x \pm r)^{2}+(y \pm \sqrt{3} r)^{2}} .
$$

Let $y_{\mathrm{i}}=d_{\mathrm{cs}^{-}}-d_{\mathrm{i}}$, then $y_{\mathrm{i}}$ determines the overlapping area of carrier sensing area and the co-channel OBSS, i.e. the spatial reuse occupy area (SOA). We then use the area of a triangle with its vertex same as the nearest vertex of co-channel OBSS, and with height $y_{\mathrm{i}}$ to approximate the SOA by ignoring the border effect. Specifically,

$$
S_{i}= \begin{cases}0 & \text { if } y_{i}<0 ; \\ \sqrt{3} y_{i}^{2} & \text { if } 0<y_{i}<\frac{r}{2} ; \\ \frac{\sqrt{3}}{4} r^{2}+\left(y_{i}-\frac{r}{2}\right) \sqrt{3} r & \text { if } \frac{r}{2}<y_{i}<\frac{3 r}{2} ; \\ \frac{3 \sqrt{3}}{2} r^{2}-\sqrt{3}\left(\frac{r}{2}-\left(y_{i}-\frac{3 r}{2}\right)\right)^{2} & \text { if } \frac{3 r}{2}<y_{i}<2 r \\ \frac{3 \sqrt{3}}{2} r^{2} & \text { if } y_{i}>2 r .\end{cases}
$$

Process similarly, let $y^{\prime}=d_{P C S}-\sqrt{x^{2}+y^{2}}$, we can get the hidden node area (HA)

$$
S_{h}=\left\{\begin{array}{lll}
\frac{3 \sqrt{3}}{4} r^{2}-\sqrt{3} r y^{\prime} & \text { if } & 0<y^{\prime}<\frac{r}{2} \\
\sqrt{3}\left(r-y^{\prime}\right)^{2} & \text { if } & \frac{r}{2}<y^{\prime}<r \\
0 & \text { if } & y^{\prime}>r .
\end{array}\right.
$$

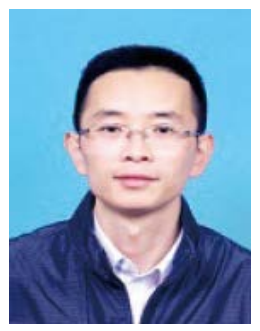

Deqing Zhu received his B.S. degree in electronic information science and technology from Shandong University, Shandong, China, in 2003; his M.S. degree in electronic science and technology from Zhejiang University, Zhejiang, China in 2010; and his Ph.D. degree in control science and engineering from Zhejiang University of Technology, Zhejiang, China in 2016. Now he is with the Academic Affairs Office, Hangzhou Normal University, Zhejiang, China. His current research interests include stochastic modeling and analysis, mobility management for wireless networks, etc. (Email:dqzhu@hznu.edu.cn).

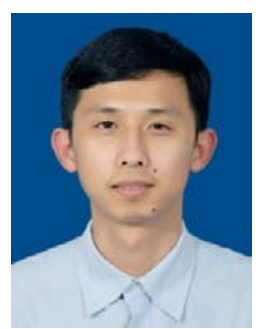

Shenji Luan received his B.S. and M.S. degrees both in Communication Engineering from Hangzhou Dianzi University, Hangzhou, Zhejiang, China, in 2002 and 2005, respectively. He received his Ph.D. degree in Control Science and Engineering from Zhejiang University of Technology, Zhejiang, China, in 2015. He is working in the Information Engineering School, Hangzhou Dianzi University. His current research interests include power management and data communications for wireless networks including wireless local area network, wireless sensor network, and cellular network 